\title{
GEOMETRICAL SOLVING OF SOME SYSTEMS OF EQUATIONS
}

\author{
Jasmina Markoska ${ }^{1)}$, Gjorgji Markoski ${ }^{2)}$
}

\begin{abstract}
We present geometrical method for solving some systems of trigonometric, exponential, logarithmic and irrational equations. The equations are transformed and solved geometrically.
\end{abstract}

Apart from systems of linear equations, for which one can always find solution, it is often difficult to find the solution of other systems. Sometimes several substitutions are required, or even various "tricks" to get to the solution.

We will present one method in which some systems of equations can be quickly and elegantly solved. The method consists of geometric representation of the equations of the system. Of course, this only works for some systems. Most often some of the variables (or expressions of variables that are part of the system) are lengths of segments or measures of angles. This is why we have to be sure that they are positive.

Now we present a few examples of using geometry to solve systems. Moreover, the given solutions are compared to other methods of solving. For some examples two solutions are given (geometric and non-geometric).

Problem 1. Let $a, b, c \geq 0$ be real numbers, such that

$$
\begin{aligned}
& b+c+6 \sqrt{b}+4 \sqrt{c}=37, \\
& a+\frac{b}{2}+5 \sqrt{a}+4 \sqrt{b}+\sqrt{a b}=\frac{321}{2}, \\
& a+\frac{c}{2}+4 \sqrt{a}+3 \sqrt{c}+\sqrt{a c}=139 .
\end{aligned}
$$

Calculate the value of $\sqrt{a b}+\sqrt{b c}+\sqrt{a c}+5 \sqrt{a}+3 \sqrt{b}+4 \sqrt{c}+11$.

Solution. We transform the given equations in the following manner. The first:

$$
b+c+6 \sqrt{b}+4 \sqrt{c}=37 \Leftrightarrow b+6 \sqrt{b}+9+c+4 \sqrt{c}+4=50
$$

2010 Mathematics Subject Classification.97D40,

Key words and phrases. Systems of equations, geometrical method 


$$
\Leftrightarrow(\sqrt{b}+3)^{2}+(\sqrt{c}+2)^{2}=50,
$$

The second:

$$
\begin{aligned}
a+\frac{b}{2}+ & 5 \sqrt{a}+4 \sqrt{b}+\sqrt{a b}=\frac{321}{2} \Leftrightarrow \\
\Leftrightarrow & a+2 \sqrt{a}+1+\sqrt{a b}+3 \sqrt{a}+\sqrt{b}+3+\frac{b+6 \sqrt{b}+9}{2}=169 \\
\Leftrightarrow & (\sqrt{a}+1)^{2}+(\sqrt{a}+1)(\sqrt{b}+3)+\frac{1}{2}(\sqrt{b}+3)^{2}=169
\end{aligned}
$$

and the third:

$$
\begin{aligned}
a+\frac{c}{2}+ & 4 \sqrt{a}+3 \sqrt{c}+\sqrt{a c}=139 \Leftrightarrow \\
\Leftrightarrow & \Leftrightarrow+2 \sqrt{a}+1+\sqrt{a c}+2 \sqrt{a}+\sqrt{c}+2+\frac{1}{2}(c+4 \sqrt{c}+4)=144 \\
\Leftrightarrow & (\sqrt{a}+1)^{2}+(\sqrt{a}+1)(\sqrt{c}+2)+\frac{1}{2}(\sqrt{c}+2)^{2}=144
\end{aligned}
$$

So, the following holds:

$$
\left\{\begin{array}{l}
(\sqrt{a}+1)^{2}+(\sqrt{a}+1)(\sqrt{b}+3)+\frac{1}{2}(\sqrt{b}+3)^{2}=169 \\
(\sqrt{b}+3)^{2}+(\sqrt{c}+2)^{2}=50 \\
(\sqrt{a}+1)^{2}+(\sqrt{a}+1)(\sqrt{c}+2)+\frac{1}{2}(\sqrt{c}+2)^{2}=144
\end{array}\right.
$$

and we need to calculate value of the expression:

$$
\begin{aligned}
\sqrt{a b}+ & \sqrt{b c}+\sqrt{a c}+5 \sqrt{a}+3 \sqrt{b}+4 \sqrt{c}+11= \\
& =\sqrt{a b}+3 \sqrt{a}+\sqrt{b}+3+\sqrt{b c}+2 \sqrt{b}+3 \sqrt{c}+6+\sqrt{a c}+\sqrt{c}+2 \sqrt{a}+2 \\
& =(\sqrt{a}+1)(\sqrt{b}+3)+(\sqrt{b}+3)(\sqrt{c}+2)+(\sqrt{c}+2)(\sqrt{a}+1) .
\end{aligned}
$$

Let $x=\sqrt{a}+1, y=\sqrt{b}+3, z=\sqrt{c}+2$. Then $x, y, z>0$ and the following holds:

$$
\left\{\begin{array}{l}
x^{2}+x y+\frac{y^{2}}{2}=169 \\
y^{2}+z^{2}=50 \\
x^{2}+x z+\frac{z^{2}}{2}=144
\end{array}\right.
$$

and

$$
(\sqrt{a}+1)(\sqrt{b}+3)+(\sqrt{b}+3)(\sqrt{c}+2)+(\sqrt{c}+2)(\sqrt{a}+1)=x y+y z+z x .
$$

To solve the system (1) we need several substitutions and transformations.

The formulation of the problem does not require us to solve the system, but only to calculate the value of the expression. Thus, without solving the system, we need to calculate value of the given expression.

This will be done using geometry. We transform the system to: 


$$
\left\{\begin{array}{l}
13^{2}=x^{2}+\left(\frac{y}{\sqrt{2}}\right)^{2}-2 x \frac{y}{\sqrt{2}} \cos 135^{\circ} \\
\left(\frac{y}{\sqrt{2}}\right)^{2}+\left(\frac{z}{\sqrt{2}}\right)^{2}=25 \\
12^{2}=x^{2}+\left(\frac{z}{\sqrt{2}}\right)^{2}-2 x \frac{z}{\sqrt{2}} \cos 135^{\circ}
\end{array} .\right.
$$

Since $x, y, z>0$, from the second equation it follows that $\frac{y}{\sqrt{2}}, \frac{z}{\sqrt{2}}, 5$ are sides of a right triangle. From the first equation and the law of cosines, it follows that $x, \frac{z}{\sqrt{2}}, 13$ are sides of triangle with angle of $135^{\circ}$ opposite of the side with length 13. Similarly, from the third equation, $x, \frac{z}{\sqrt{2}}, 12$ are sides of triangle with angle of $135^{\circ}$ opposite of the side with length 12 .

This situation is given on the figure.

The triangle $A C B$ is a right triangle.

We have:

$$
\begin{aligned}
& P_{\triangle A D B}=\frac{1}{2} x \frac{y}{\sqrt{2}} \sin 135^{\circ}=\frac{1}{4} x y, \\
& P_{\triangle A D C}=\frac{1}{2} x \frac{z}{\sqrt{2}} \sin 135^{\circ}=\frac{1}{4} x z, \\
& P_{\triangle B D C}=\frac{1}{2} \frac{y}{\sqrt{2}} \frac{z}{\sqrt{2}}=\frac{1}{4} y z,
\end{aligned}
$$

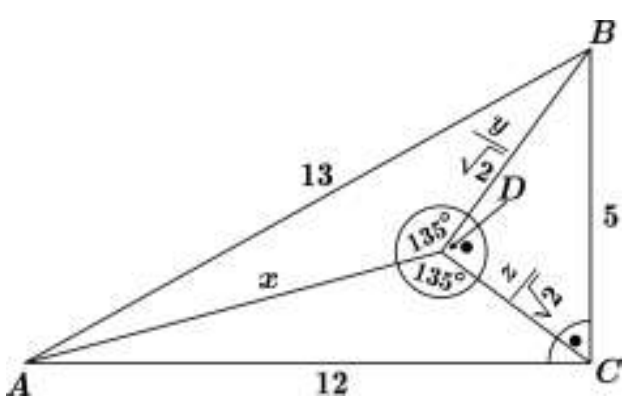

so:

$$
x y+y z+x z=4\left(P_{\triangle A D B}+P_{\triangle A D C}+P_{\triangle B D C}\right)=4 P_{\triangle A B C}=\frac{1}{2} \cdot 12 \cdot 5=30 .
$$

Problem 2. Solve the system:

$$
\left\{\begin{array}{l}
\ln ^{2} x+4 \ln x+y \ln x+2 y+y^{2}=0 \\
\ln ^{2} x+5 \ln x+e^{z} \ln x+4 e^{z}+e^{2 z}=2 \\
y^{2}+y e^{z}+y+e^{2 z}+2 e^{z}=35
\end{array}\right.
$$

where $x>1, y>0$ and $z \in \mathbb{R}$.

Solution. We transform the equations in the following manner: the first

$$
\begin{aligned}
\ln ^{2} x+4 \ln x+y \ln x+2 y+y^{2}=0 & \Leftrightarrow \ln ^{2} x+4 \ln x+4+y \ln x+2 y+y^{2}=4 \\
& \Leftrightarrow(\ln x+2)^{2}+y(\ln x+2)+y^{2}=4
\end{aligned}
$$

the second

$$
\ln ^{2} x+5 \ln x+e^{z} \ln x+4 e^{z}+e^{2 z}=2 \Leftrightarrow
$$




$$
\begin{aligned}
& \Leftrightarrow \ln ^{2} x+4 \ln x+4+e^{z} \ln x+\ln x+2 e^{z}+2+e^{2 z}+2 e^{z}+1=9 \\
& \Leftrightarrow(\ln x+2)^{2}+(\ln x+2)\left(e^{z}+1\right)+\left(e^{z}+1\right)^{2}=9
\end{aligned}
$$

and the third

$$
y^{2}+y e^{z}+y+e^{2 z}+2 e^{z}=35 \Leftrightarrow y^{2}+y\left(e^{z}+1\right)+\left(e^{z}+1\right)^{2}=36
$$

So the given system is transformed to the equivalent system:

$$
\left\{\begin{array}{l}
(\ln x+2)^{2}+y(\ln x+2)+y^{2}=4 \\
(\ln x+2)^{2}+(\ln x+2)\left(e^{z}+1\right)+\left(e^{z}+1\right)^{2}=9 \\
y^{2}+y\left(e^{z}+1\right)+\left(e^{z}+1\right)^{2}=36
\end{array}\right.
$$

Now, we substitute $a=\ln x+2, b=y$ and $c=e^{z}+1$. Thus:

$$
\left\{\begin{array}{l}
a^{2}+a b+b^{2}=4 \\
a^{2}+a c+c^{2}=9 \\
b^{2}+b c+c^{2}=36
\end{array}\right.
$$

Since $x>1, y>0$ and $z \in \mathbb{R}$, it follows that $a, b, c>0$, so we can use geometry to solve the system (2). We transform the system in the following manner:

$$
\left\{\begin{array}{l}
2^{2}=a^{2}+b^{2}-2 a b \cos 120^{\circ} \\
3^{2}=a^{2}+c^{2}-2 a c \cos 120^{\circ} \\
6^{2}=b^{2}+c^{2}-2 b c \cos 120^{\circ}
\end{array}\right.
$$

From the law of cosines, it follows that $a$ and $b$ are sides of a triangle and that the angle between $a$ and $b$ is $120^{\circ}$, while side opposite to it is 2 . Also, $a$ and $c$ are sides of a triangle with angle of $120^{\circ}$ between them, while the third side equals 3 , and $b$ and $c$ are sides of a triangle with angle between them $120^{\circ}$, while the third side equals to 6 . So, we obtain the figure, i.e. there exists a triangle with sides $2,3,6$, which is not possible. We conclude that there are no real positive numbers $a, b, c$ such that the given equations hold, so the initial system does not have a solution.

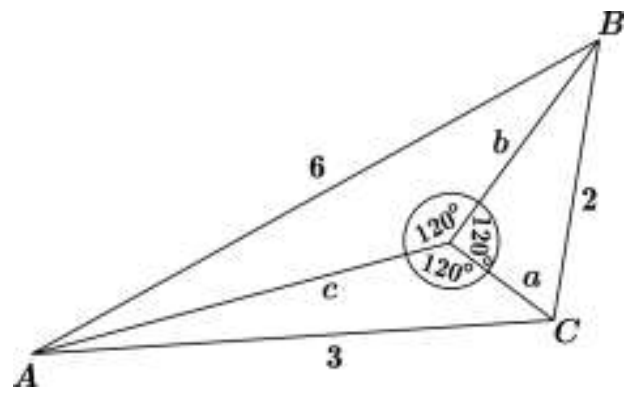

Remark. If we substract the second equation from the first in (2) we get: 


$$
a(b-c)+b^{2}-c^{2}=-5 \text {, i.e. }(b-c)(a+b+c)=-5 .
$$

Since $a+b+c>0$, it follows that $b-c<0$, i.e. $b<c$. Simillary, $a<b<c$.

From the first equation it follows that $4=a^{2}+a b+b^{2}<3 b^{2}$, so $b>\frac{2}{\sqrt{3}}$, and from the third equation we get that $c>\sqrt{12}$. From the second equation it follows that

$$
9=a^{2}+a c+c^{2}>a^{2}+\sqrt{12} a+12, a^{2}+\sqrt{12} a+3<0 \text {, i.e. }(a+\sqrt{3})^{2}<0,
$$

which is not possible.

Problem 3. Solve the system:

$$
\left\{\begin{array}{l}
(2 \sin a-\cos b) \sqrt{\sin a \cos b}=\frac{3}{2 \sqrt{2}} \\
2 \sin a+\sqrt{2 \sin a \cos b}=3
\end{array}\right.
$$

if $a \in[0, \pi]$ and $b \in\left[-\frac{\pi}{2}, \frac{\pi}{2}\right]$.

Solution. If $a=0$, or $a=\pi$, or $b=-\frac{\pi}{2}$, or $b=\frac{\pi}{2}$, then the first equation does nod holds, so those numbers are not solution of the system. Let $a \in(0, \pi)$ and $b \in\left(-\frac{\pi}{2}, \frac{\pi}{2}\right)$, i.e. $\sin a>0$ and $\cos b>0$. We transform the first equation in the following manner:

$$
\begin{aligned}
& (2 \sin a-\cos b) \sqrt{\sin a \cos b}=\frac{3}{2 \sqrt{2}} \Leftrightarrow 2(2 \sin a-\cos b) \sqrt{2 \sin a \cos b}=3 \\
& \quad \Leftrightarrow 32(2 \sin a-\cos b) \sqrt{2 \sin a \cos b}=48 \\
& \quad \Leftrightarrow 8(8 \sin a-4 \cos b) \sqrt{2 \sin a \cos b}=48 \\
& \Leftrightarrow(8 \sin a-4 \cos b) \sqrt{128 \sin a \cos b}=48 \\
& \Leftrightarrow(8 \sin a-4 \cos b) \sqrt{(8 \sin a+4 \cos b)^{2}-(8 \sin a-4 \cos b)^{2}}=48
\end{aligned}
$$

The second:

$$
\begin{aligned}
& 2 \sin a+\sqrt{2 \sin a \cos b}=3 \Leftrightarrow 16 \sin a+\sqrt{128 \sin a \cos b}=24 \Leftrightarrow \\
& \Leftrightarrow(8 \sin a+4 \cos b)+(8 \sin a-4 \cos b)+\sqrt{(8 \sin a+4 \cos b)^{2}-(8 \sin a-4 \cos b)^{2}}=24 .
\end{aligned}
$$

Thus, the initial system is equivalent to the system:

$$
\left\{\begin{array}{l}
(8 \sin a-4 \cos b) \sqrt{(8 \sin a+4 \cos b)^{2}-(8 \sin a-4 \cos b)^{2}}=48 \\
(8 \sin a+4 \cos b)+(8 \sin a-4 \cos b)+\sqrt{(8 \sin a+4 \cos b)^{2}-(8 \sin a-4 \cos b)^{2}}=24 .
\end{array}\right.
$$

We substitute $x=8 \sin a+4 \cos b$ and $y=8 \sin a-4 \cos b$. Then: 


$$
\left\{\begin{array}{l}
y \sqrt{x^{2}-y^{2}}=48 \\
x+y+\sqrt{x^{2}-y^{2}}=24
\end{array}\right.
$$

Since $\sin a>0$ and $\cos b>0$, it follows that $x=8 \sin a+4 \cos b>0$. Also, $x=8 \sin a+4 \cos b>8 \sin a-4 \cos b=y$. The first equation must hold, so $y>0$. Now we solve the system (3). It is possible to consider problem from geometric perspective. From $y^{2}+\left(\sqrt{x^{2}-y^{2}}\right)^{2}=x^{2}$, it follows that $y$ and $\sqrt{x^{2}-y^{2}}$ are legs in a right triangle, and $x$ is the hypotenuse.

From the first equation in (3), it follows that the area of that triangle is 24 , and from the second equation we get that the perimeter is 24 . Let $r$ be the radius of the circle inscribed in the triangle, $P$ be its area and $L$ its perimeter. From $P=\frac{L}{2} r$, it follows that $r=2$. In a

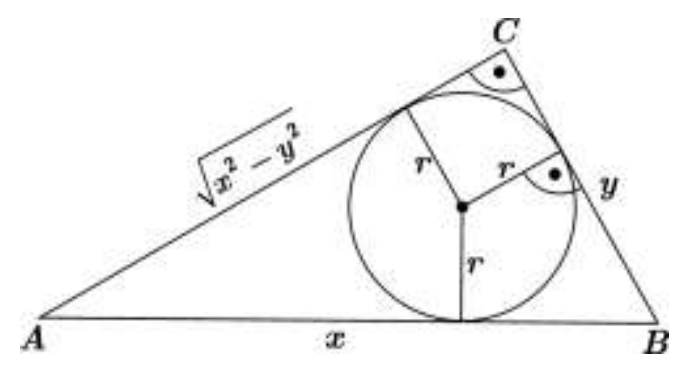
right triangle, the following holds:

$$
\begin{aligned}
a+b-2 r & =a+b-2 \frac{2 P}{L}=a+b-4 \frac{\frac{a b}{2}}{a+b+c}=a+b-\frac{2 a b}{a+b+c} \\
& =\frac{a^{2}+a b+a c+b a+b^{2}+b c-2 a b}{a+b+c}=\frac{c^{2}+a c+b c}{a+b+c}=c .
\end{aligned}
$$

Thus we get:

$$
x=y+\sqrt{x^{2}-y^{2}}-2 r=y+\sqrt{x^{2}-y^{2}}-4 .
$$

From the second equation, it follows that $x+x+4=24$, i.e. $x=10$. From first equations we obtain two values for $y, y_{1}=6$ and $y_{2}=8$.

If $x=10$ and $y=6$ then

$$
\left\{\begin{array}{l}
8 \sin a+4 \cos b=10 \\
8 \sin a-4 \cos b=6 .
\end{array}\right.
$$

The solution is $\sin a=1$ i.e. $a=\frac{\pi}{2}$ and $\cos b=\frac{1}{2}$ i.e. $b=\frac{\pi}{3}$ or $b=-\frac{\pi}{3}$.

Solutions of the initial system are $\left(\frac{\pi}{2}, \frac{\pi}{3}\right)$ and $\left(\frac{\pi}{2},-\frac{\pi}{3}\right)$.

If $x=10$ and $y=8$ we obtain the system

$$
\left\{\begin{array}{l}
8 \sin a+4 \cos b=10 \\
8 \sin a-4 \cos b=8 .
\end{array}\right.
$$


It follows that $\sin a=\frac{18}{16}$, which is not possible.

Finally solutions of the system are $\left(\frac{\pi}{2}, \frac{\pi}{3}\right)$ and $\left(\frac{\pi}{2},-\frac{\pi}{3}\right)$.

Remark. With the substitution $\sqrt{x^{2}-y^{2}}=\frac{48}{y}$ in second equation, we can express $x$ through $y$, and then the expression for $x$ is substituted in the first equation. Then we only need to solve one equation in one variable. However, this solution require a lot of calculations.

Problem 4. Solve the equation $\sqrt{4 \sin ^{2} z+5}+\sqrt{9 \cos ^{2} z-5}=5$.

Solution. We can solve this equation in a "standard" way by squaring both sides. Here we solve the equation geometrically. First, we notice that the equation is defined for those $z \in \mathbb{R}$ such that $\cos ^{2} z>\frac{5}{9}$. We substitute $x=2 \sin z$ and $y=3 \cos z$. Then $x^{2}+y^{2}=13$, and we obtain the system

$$
\left\{\begin{array}{l}
x^{2}+y^{2}=13 \\
\sqrt{x^{2}+5}+\sqrt{y^{2}-5}=5
\end{array}\right.
$$

We can solve this system by method of substitution. Expressing $x^{2}$ from the first equation, then substituting for it in second equation, and then squaring the obtained equation, we will get the solution for the system.

Let us consider a geometric solution. First, if a pair $(x, y)$ is a solution of a system, then $(-x,-y),(x,-y)$ and $(-x, y)$ are also solutions. Since $\cos ^{2} x \leq 1$, it follows that $y=3 \cos ^{2} z \neq 5$. Further,

$$
\left(\sqrt{x^{2}+5}\right)^{2}+\left(\sqrt{y^{2}-5}\right)^{2}=x^{2}+y^{2}=\sqrt{13}^{2},
$$

so we obtain that the positive numbers $\sqrt{x^{2}+5}$, $\sqrt{y^{2}-5}$ and $\sqrt{13}$ are sides of a right triangle.

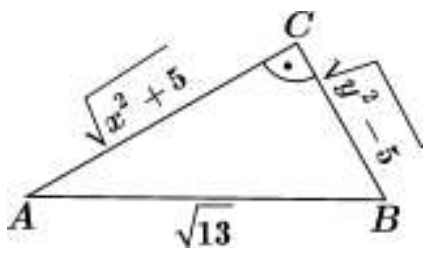

Let $a$ and $b$ be the legs, and $c$ the hypotenuse of that right triangle, then $a+b=5$ and $a^{2}+b^{2}=13$. By substitution we obtain the equation $a^{2}+(5-a)^{2}=13$. Solutions of this equation are $a_{1}=2$ and $a_{2}=3$, from where $b_{1}=3$ and $b_{2}=2$. This means that $\sqrt{x^{2}+5}=2$ or $\sqrt{x^{2}+5}=3$.

Since $\sqrt{x^{2}+5} \geq \sqrt{5}>2$, we conclude that $\sqrt{x^{2}+5}=2$ is not possible. 
Thus $\sqrt{x^{2}+5}=3$, so $x=2$ or $x=-2$, from where $y=3$ or $y=-3$. Solutions of a system are the pairs $(2,3),(-2,-3),(-2,3)$ and $(2,-3)$.

Therefore we get the following cases:

1) $2 \sin z=2,3 \cos z=3$, i.e. $\sin z=1$ and $\cos z=1$.

2) $2 \sin z=-2,3 \cos z=-3$, i.e. $\sin z=-1$ and $\cos z=-1$.

3) $2 \sin z=-2,3 \cos z=3$, i.e. $\sin z=-1$ and $\cos z=1$.

4) $2 \sin z=2,3 \cos z=-3$, i.e. $\sin z=1$ and $\cos z=-1$.

None of these cases is possible, so the equation has no solutions.

Remark. Since

$$
\sqrt{4 \sin ^{2} z+5} \leq \sqrt{4+5}=3 \text { and } \sqrt{9 \cos ^{2} z-5} \leq \sqrt{9-5}=2
$$

we obtain $\sqrt{4 \sin ^{2} z+5}=3$ and $\sqrt{9 \cos ^{2} z-5}=2$. This holds only for $\sin z \in\{-1,1\}$ and $\cos z \in\{-1,1\}$. However, that is not possible, so the initial equation has no solutions.

This methods is, of course, much shorter, but because of the connection to a geometric situation, it is useful to present a geometric method.

Problem 5. Solve the system of equations

$$
\left\{\begin{array}{l}
6 \sin a+6 \cos a+3 \sqrt{b^{2}-3}+5=0 \\
12 \sin a+84 \cos a+9 b^{2}+56=0
\end{array}\right.
$$

Solution. Obviously $b^{2} \geq 3$.

We will transform the first equation as follows:

$$
\begin{aligned}
6 \sin a+6 \cos a+3 \sqrt{b^{2}-3}+5=0 & \Leftrightarrow 2 \sin a+2 \cos a+\sqrt{b^{2}-3}+\frac{5}{3}=0 \\
& \Leftrightarrow 2 \sin a+\frac{1}{3}+2 \cos a+\frac{7}{3}+\sqrt{b^{2}-3}=1
\end{aligned}
$$

and a second equation:

$$
\begin{aligned}
12 \sin a & +84 \cos a+9 b^{2}+56=0 \Leftrightarrow \frac{4}{3} \sin a+\frac{28}{3} \cos a+b^{2}+\frac{56}{9}=0 \\
& \Leftrightarrow 4+\frac{4}{3} \sin a+\frac{28}{3} \cos a+b^{2}-3+\frac{47}{9}=0 \\
& \Leftrightarrow 4\left(\sin ^{2} a+\cos ^{2} a\right)+\frac{4}{3} \sin a+\frac{28}{3} \cos a+b^{2}-3+\frac{47}{9}=0 \\
& \Leftrightarrow 4 \sin ^{2} a+\frac{4}{3} \sin a+\frac{1}{9}+4 \cos ^{2} a+\frac{28}{3} \cos a+\frac{49}{9}+b^{2}-3-\frac{3}{9}=0 \\
& \Leftrightarrow\left(2 \sin a+\frac{1}{3}\right)^{2}+\left(2 \cos a+\frac{7}{3}\right)^{2}+\left(\sqrt{b^{2}-3}\right)^{2}=\frac{1}{3} .
\end{aligned}
$$

We substitute 


$$
x=2 \sin a+\frac{1}{3}, y=2 \cos a+\frac{7}{3} \text { and } z=\sqrt{b^{2}-3} .
$$

We obtain:

$$
\left\{\begin{array}{l}
x+y+z=1 \\
x^{2}+y^{2}+z^{2}=\frac{1}{3}
\end{array}\right.
$$

Let $\vec{s}=(x, y, z), \vec{t}=(1,1,1)$ be vectors in space.

Then:

$$
|\vec{s}|=\sqrt{x^{2}+y^{2}+z^{2}}=\sqrt{\frac{1}{3}} \text { and }|\vec{t}|=\sqrt{1^{2}+1^{2}+1^{2}}=\sqrt{3} .
$$

The scalar product of the vectors $\vec{s}$ and $\vec{t}$ is $\vec{s} \cdot \vec{t}=x \cdot 1+y \cdot 1+z \cdot 1$. From the first equation of the system we obtain $\vec{s} \cdot \vec{t}=1$, and $|\vec{s}| \cdot|\vec{t}|=\sqrt{\frac{1}{3}} \cdot \sqrt{3}=1$. This means that $\vec{s} \cdot \vec{t}=|\vec{s}| \cdot|\vec{t}|$, from were $\cos \Varangle(\vec{s}, \vec{t})=1$.

The vectors are collinear and they have the same direction. So, there exists $k \in \mathbb{R}$ such that $\vec{a}=k \cdot \vec{b}$, i.e. $(x, y, z)=k(1,1,1)$. If $k<0$ vectors have opposite directions, i.e. $\cos \Varangle(\vec{s}, \vec{t})=-1$. If $k=0$, then $\vec{s}=\overrightarrow{0}$. This is not possible because $|\vec{s}|=\sqrt{\frac{1}{3}}$. This means that $k>0$.

From there we obtain, $x=k, y=k, z=k$, and from the first equation in the system we have that $k=\frac{1}{3}$. Thus, the solution of the system (1) is $(x, y, z)=\left(\frac{1}{3}, \frac{1}{3}, \frac{1}{3}\right)$.

Now, we obtain $2 \sin a+\frac{1}{3}=\frac{1}{3}, 2 \cos a+\frac{7}{3}=\frac{1}{3}$ and $\sqrt{b^{2}-3}=\frac{1}{3}$, so $\sin a=0$, $\cos a=-1$ and $b=\frac{2 \sqrt{7}}{3}$.

Finally, $a=2 m \pi, m \in \mathbb{Z}$ and $y=\frac{28}{9}$ is the solution of the system.

Remark 1. If we substitute $x=1-y-z$ from the first equation of the system (1) to the second equation, we obtain $y^{2}-(1-z) y+z^{2}-z+\frac{1}{3}=0$. The quadratic equation in $y$ has real solutions if and only if $D=-3 z^{2}+2 z-\frac{1}{3} \geq 0$. This inequality holds if and only if $z=\frac{1}{3}$. Substituting $z=\frac{1}{3} \quad$ in $y^{2}-(1-z) y+z^{2}-z+\frac{1}{3}=0$, we obtain $y^{2}-\frac{2}{3} y+\frac{1}{9}=0$, i.e. $\left(y-\frac{1}{3}\right)^{2}=0$. So $y=\frac{1}{3}$ and $x=\frac{1}{3}$. 
Remark 2. Geometrically, the system (1) represents the intersection of the plane $x+y+z=1$ and the sphere $x^{2}+y^{2}+z^{2}=\frac{1}{3}$. Since the radius of the sphere is $\frac{1}{\sqrt{3}}$ and the distance from the center to the plane is $\frac{1}{\sqrt{3}}$, it follows that the plane is tangent to the sphere, so there is only one intersection point $(x, y, z)=\left(\frac{1}{3}, \frac{1}{3}, \frac{1}{3}\right)$.

\section{References}

[1] Г. 3. Генкин, Геометрические решения негеометрических задач, Москва, 2007.

[2] Textbooks in mathematics for second and third year of high school

[3] J. Markoska, Gj. Markoski, Geometrical solving of systems of equations I, Sigma 91, 2010/2011, 8-13 (in macedonian)

[4] J. Markoska, Gj. Markoski, Geometrical solving of systems of equations II, Sigma 92, 2010/2011, 1-5 (in macedonian)

1) SUGS "Georgi Dimitrov", Skopje,

R. Macedonia

jmarkoska@gmail.com
2) Faculty of Natural Sciences and Mathematics Ss. Cyril and Methodius University, Skopje, R. Macedonia gorgim@pmf.ukim.mk 